\title{
Promoting Mothers' Psychological Health after Fetal Loss-Descriptive Case Reports
}

\author{
Shaheena Salman Alwani* \\ Assistant Manager OPD Services, Liaquat National Hospital and Medical College, Pakistan
}

Submission: May 25, 2017; Published: June 05, 2017

*Corresponding author: Shaheena Salman Alwani, Assistant Manager OPD Services, Liaquat National Hospital and Medical College, Pakistan, Tel: +923333316730; Email: Shaheena.salman@Inh.edu.pk

\section{Background/Objective}

Life is the biggest gift of God and the process of giving birth is praise-worthy. Therefore, maternal mortality and morbidity is alarming and need extensive interventions. The psychosocial and psychological perspectives of childbirth need attention and vigorous trainings for health- care workers. Many countries are working to improve standards of their living and to curtail subsequent life threatening illness associated with pregnancy and birth. For that, different reporting, evaluating and practicing guidelines made to check the present practices that have greater impact on health-care industries and on patients. One of the widely eminent is CEMACH (Confidential Enquiry into Maternal and Child Health). According to Weindling [1], CEMACH was established in April 2003. Previously it worked as CESDI (the Confidential Enquiry into Stillbirths and deaths in infancy) and CEMD (the Confidential Enquiry into Maternal Deaths). Formerly, the ministry of health had not reported adequately and timely about the maternal deaths but it started its formalized reporting in the year 1952. In the first 2 years of CEMD, about $77 \%$ of maternal deaths were captured and later for the United Kingdom, in years 1985-87 onwards reports were published. CEMD's motive was to assess the causes of maternal deaths and to indentify avoidable causes to reduce it. It recommended improvements in clinical setup and care provision. Moreover, future research and way forward to audits were indicated. Later, it was facilitated by department of health that all 14 regions of England should embark on perinatal mortality survey reports and thus CESDI, established in 1992, aimed to reduce mortality by finding practice gaps and its improvement especially cases that encountered less than 20 weeks post-conception to 1 year of birth. Confidential enquiries aim was to improve practices and care. Weindling [1] reported that six permanent staff with allotted managers were assigned in each of the regions of England and Wales. Further, the United Kingdom's MMR (maternal mortality rate) can be determined by routine death-certificate data and by the results of in-depth enquiry. CMACE [2] illustrates that the use of routine death-certificate data is consistently under-reported therefore; the most accurate numbers are acquire via Enquiry.

CMACE [2] affirms that the UK's trends of maternal deaths have fallen over the last 3 years. At the same time, women dying from infections have also been noted. CMACE [2] states: "The MMR was 11.39 per 100,000 maternities compared to 13.95 per 100,000 maternities for the previous triennium, 2003-05. As this enquiry is far more inclusive than in other countries, for direct comparison with international figures, the UK maternal death rate was 6.7 per 100,000 live births." Moreover, the report illustrates that 261 women in the UK died from conditions directly or indirectly related to pregnancy in the year 20062008, out of which 107 were direct deaths and remaining 154 died of other associated causes. The direct death rate decreased from 6.24 to 4.67 per 100,000 maternities. The report evokes pregnant women and hospital staff to be sentient of the need for meticulous hygiene during and after birth and contact of a mother with others having sore throats. CMACE [2] also reminds for national guidelines pertaining to sepsis management and identification in delivered and pregnant women; developing countries, health status and government interest needs thorough intervention; developed and under-developed countries must focus on preventing maternal morbidities and mortalities. According to WHO [3], Under-developed country- Pakistan's MMR in year 2008 was 260/100,000 live births. Substantially, many guidelines and protocols such as RCOG, NICE are adapted by Pakistani hospitals. However, not all hospitals have incorporated these in clinical practices. Some private hospitals of Pakistan, adapted Obs/Gyne guidelines considering CEMACH, NICE and RCOG for providing optimum level of care. In addition, maternal mortality and morbidity meetings and educational sessions are core components at AKUH-P's Obs/Gyne department. On the other hand, government based hospitals require facilitation and boost. Quality of life and care are the components that need facilitation through a strong influential government. 


\section{Global Journal of Reproductive Medicine}

Moreover, the era for proactive approaches in order to subsidize standard care, needs expert personnel and institutions. Not only shortage of nurse-midwives and physicians can contribute to sub-standard care; lack of supervisory skills, facilitators, trainers, multi-functional teams can add up to the burden. AKUH-P, has strived to maintain the standards via resourced, skilled maternity work force and clinical affairs department that scrutinizes, investigates root causes for incidents and sentinel events. This guides the department for corrective and preventive approaches and deals with the complex issues. In order to have such kind of teams in government based hospitals, authorities must provide facilities to the health-care workers such as appropriate salaries, on-job trainings, medical benefits and allowances. This will help in achieving the fifth millennium development goal. WHO [3] shares that it aim to reduce MMR by $75 \%$ between 1990 and 2015. It seeks to achieve a 5.5\% annual decline in MMR from 1990. Globally, the annual percentage decline in MMR between 1990 and 2008 was 2.3\%. This can improve much, if we can take above-mentioned steps in both oriental and occidental societies.

\section{Methods}

Here the review of trends of causes of death in UK is worth noticing. Firstly, it is obvious that deaths due to sepsis are increasing. It was 13 in the year 2000-02, which increased up to 18 in numbers in 2003-05, subsequently increased up to 26 in years 2006-08. Similarly, deaths from preeclampsia and eclampsia are also escalating i.e. 14, 18 and 19 in years 2000 02, 2003-05 and 2006-08 respectively. At the same time, deaths from spontaneous miscarriage were sustained in years 20002005 i.e. 1 but significantly increased up to 5 in years 200608. Adding, deaths from anaesthesia remained almost same with the difference of 1 number that increased in year 200608 , which were 6 in 2 previous reports. Indirect cardiac causes found increasing too. The indirect cause of death described as psychiatric causes, that were reported 13 in numbers from years 2006-08, 18 in numbers from years 2003-05 and 16 in numbers from years 2000-02 and through CMACE [2] review; psychiatric causes led to this. On the other hand, deaths from indirect neurological conditions is significant but have dropped too from 40 in numbers in years 2000-02 to 37 in years 2003-05 and 36 in numbers in 2006-08 years. Moreover, deaths from psychiatric causes fluctuated from numbers 16 to 18 and then 13 in three consecutive reports.

Out of all psychiatric causes, fetal loss and bereavement is the area of importance. It may contribute to suicidal attempts if not treated earlier. Fetal loss in terms of Termination of pregnancy (TOP), therapeutic abortion, fetal demise, miscarriages, fetal anomalies, neuro-muscular abnormalities etc. is pain full to admit. CEMACH [4] discusses 3 cases; that killed themselves following medical TOP. Previously, no suicide was reported following TOP. Similarly, through CMACE [2] 2 deaths associated with TOP were due to staphylococcal toxic shock syndrome, Clostridium septicum and non-prescription of prophylactic antibiotics in both cases, and not because of suicide. However, via CMACE [2] review; 8 women were admitted to psychiatric units and out of those; 1 was admitted following TOP. If we analyse suicide rates, CMACE [2] depicts number of maternal deaths from suicide in UK that had increased in numbers from 20 to 29 during years 2003-2005 to 2006-2008. Noticeably, it was 22 in numbers in years 2000-2002. Out of these 29-recorded deaths within the time range of 2006-2008; 3 of them died of suicide due to nonadjustment/ grief reactions and 2 out of these 3 were followed after the loss of early pregnancy. Thus, correlation of suicide and fetal loss is evident and a need of concern for developed as well as under-developed countries. The relation of fetal loss and bereavement is discussed in detail later in this essay. The role of professionals in helping the families going through bereavement, grief and mourning is pivotal. Thus, it is fundamental to practice standard care guidelines.

An alive and healthy newborn cherishes the family. However, horrendous news of losing a baby, impairs mental health that is incomprehensible, not only parents are in despair but also family suffers the irreparable loss. Perinatal loss is an anticipated loss of parenthood with loss of all images associated with future. The mother not only loses pregnancy but the experience of childbirth, holding her newborn and nursing it. When foetus is loss; the future is loss, which includes mother's delivery process, seeing baby grow, dreams attached with the baby like graduation, wedding; the delirious moments turn into bereavement. Complexity increases when termination occurs on medical ground, either maternal morbidity/mortality is a concern or baby's congenital abnormalities. This dreadful incident has detrimental effect on mothers' mental health. As professional, it is necessary to know the consequences of losing a baby, which certainly affects the soul and mind of mothers. CLIMB [5] discussed 'group arrival' of babies, as extra blessings and special prestige among families and communities and death of twin or triplets can be depressing for women. Depressive illness can be found amongst those who losses the baby after having infertility treatments or those who have difficulty in conceiving. All these women experience grief upon losing whether, they are primi or multi-gravida.

Kübler-Ross, in the year 1969 identified 5 stages of grief i.e.: denial, anger, bargaining, depression and acceptance. These stages are non- sequential and may vary person to person. In study, Hoy [6] illustrates that every individual is different in adaptation and vary in reactions towards loss and should not be termed as abnormal grieving if any stage is missing. Moreover, impact of society and professional guidance help women to overcome grief. In prospective longitudinal study, Nikcevic et al. [7] investigated and informed that knowledge of the possible cause of miscarriage reduces women's long-term psychological distress. The designed research questioner identified anxiety, self-blame, grief and depression at 4 weeks and at 4 th month of fetal loss. It clinically revealed that elevated levels of anxiety 


\section{Global Journal of Reproductive Medicine}

were found in $45 \%$ of women and depression in $15 \%$ of them at 16-400 days after diagnosis of a missed miscarriage. Subsequently, the levels of grief and psychological morbidity were high. It reflected that scores for all outcome-variables were lower in the four-week post-miscarriage women. Therefore, it is presume that intensity reduces with passage of time. Further, when women are informed prior regarding fetal abnormalities, reports significantly less self-blame than those in whom no cause identified earlier [7]. Therefore, such a research is step forward to assist relieving grief and a clinical tool to know the importance of informed treatment regimen and patient education.

On the other hand, if professional guidance is sub-optimal, chances for developing psychiatric illness are higher. It is hypothetical that it may be the cause or effect of loss and bereavement that mothers under-go into severe mental illness, which leads to issues related to psychosocial, psychological, physiological, spiritual discordant, fetal loss and suicide. DHSSPS [8] shared that traumatic events may lead to variety of related symptoms including Post-traumatic stress disorder and other mental-health disorders e.g. depression, phobic anxiety and self-harm. Protect life [9] explicates that today's sensitive and poignant matter is suicide that can be due to depression, alcohol and drug ingestion, personality disorder, bereavement, social isolation, state of despondency, shattered self-esteem and broken relationships. Hence, the strong relation of bereavement and mental health consequences are apparent and require interventions before it aggravates and be a cause of suicide.

The discussion over here is that an attachment is the key factor in any relationship, when successful transition to parenthood hinders; family experiences despair and chaotic bonding. It is vital to know how these assessments are carried out on women, in order to gather baseline data for further clinical interventions and to help them progressing through life. Many hospitals have acquired different 'mental status examination scales' and it is elementary to get some in practice. In study, Ritsher [10] found that PBGS (Perinatal Bereavement Grief Scale) was designed to measure grief following reproductive loss. The bereavement reactions can be self-blame, loss of appetite, anger, social seclusion, disbelief, neuro-endocrine disturbances and panic syndromes. That can be classified as

a) Shock, numbness,

b) Yearning for and preoccupation with the deceased and

c) Depression, disorganization and anxiety.

Traumatic grief comprises of a syndrome distinct both from grief and post-traumatic stress disorder, with stressor explicitly identified as traumatic loss of an attachment. Further, debate and association towards risk for suicide and progressing towards psychiatric illness are supported by many researchers in this context. These assessment scales, are eventually fruitful in identifying level of psychiatric involvement after losing pregnancy.
Health-care professionals can utilize recognized scales in order to help the victim heal. In addition, knowledge about these scales and its implementation can improve existing care practices. For this, another 4 basic scales will be discussed here. Ritsher [10] shared Potvin, Lasker \& Toedter's (1989) PGS (Perinatal Grief Scale) that comprises of 3 subscales: active grief, difficult coping and despair. Few examples where women might share depressive outcomes towards future come under 'active grief', suicidal ideation or attempt falls under 'difficulty in coping' and feeling of worthlessness comes under 'despair'. The progression of these categories can be seen more advanced and improved in PBGS scale. Only PGS can cover few aspects such as 'crying for or missing the baby and having painful memories of the losses'. On the other hand, PBGS is a 15-item scale, which is ensuing from a review of clinical, theoretical, counselling and research literature. For instance, few questions from the scale are "you dreamed you were still pregnant and you patted or held your belly as though you were still pregnant" etc. Through the literature search, it was found, that different cultures have diverse range of stances and behaviours for mother-hood, fetal demise and coping measures [10]

The other scale that can be replicated as per hospital setting is CES-D (Centre for Epidemiological Studies- Depression Scale). Ritsher [10] describes CES-D as a scale for measuring affective, cognitive and somatic symptomatology of depression comprising of 20-items asking about the presence and duration of symptoms in the preceding 7 days. Third scale, which Ritsher [10] shared, contains items response that requires true of false, based on 15-items of Crowne Marlowe's Social Desirability Scale. High scores indicate respondent's analytically appropriate response. As a health-care professional, it is to explore that whether woman thinks she has lost the baby, an embryo or just blood or tissue. Has she already named the baby, has she purchased items necessary for the baby? The cognition can be a predictor for woman's attitude towards loss. Fourthly, "Wantedness Scale by Neugebauer" as described in the study of R Ritsher [10], measures the degree to which the pregnancy had been wanted. Scores for this scale ranges from 0-12 (zero considered as unhappy to conceive). This may enable healthcare professionals that the woman is at high risk for developing emotional instability, affecting activity of daily living.

The above-described scales can be a part of protocol at health-care setting. There, the base line data for women undergoing bereavement process are gathered and given referrals to seek expert consultations. This helps the families to adjust, cope and avoid suicidal attempts. Hospitals can adapt these scales in order to assess the extent of mental illness and for relating it with care-regimen. Obviously, bereavement is a subjective theme; appropriate use of scales can be beneficial. It is the contention that fetal loss has negative influence on women's health. At the same time, reason for fetal loss can be persistent depression, anxiety and stressful life event. We cannot segregate much as to depression or anxiety is the cause of fetal loss or an 


\section{Global Journal of Reproductive Medicine}

effect of it. In any case, women needs appropriate treatment. Le \& Boyd [11] illustrated that perinatal depression is the second greatest burden on world. Mothers also experience significant levels of depressive symptomatology during pregnancy and post-partum period. Depression, at the diagnostic and symptom level, during childbearing years has found to be injurious and have negative consequences. Moreover, perinatal depression is associated with co-morbid psychopathology. This can due to be poor social-support, distorted marital relationship, or any obstetrical complication. The Edinburg Postnatal Depression Scale is most widely used scale to prevent the deleterious effects of distorted psychology and for intervening accordingly. It measures emotional and cognitive symptoms; excluding somatic symptoms associated with post-partum depression. Le \& Boyd [11] in research shared literature, which suggests, that post-partum depression can have depression onset before delivery. It further illustrates that $11.5 \%$ of women reported depressive episode's onset during pregnancy, 66.5\% during the early post-partum period and $22 \%$ in later time. Glavin [12] correlates postnatal depression and loss of pregnancy by utilizing this in research stating: "post natal depression can develop in primiparous and multiparous women. Risk factors are previous history of depression, stressful events during past years, pregnancy complications, marital conflict..." It reflected immensely in NHS [13] that nearly one month after loss; about $33 \%$ of bereaved women have symptoms of depression and $15 \%$ remains depressed after a year later too. On the contrary, supportive friends and family can help in healing and feelings may shade-off gradually.

\section{Results}

It is essential to discuss management and treatmentregimens that require skilled personnel to deal with. These are time consuming, at the same time, can bring positive changes in some one's life and may help to rejoice. Isis [14] shared that few bereaved persons have discovered that they could find solace from shattering and cutting stained glass to create multiple art objects. Despite a strong desire at times to slit wrist, picking up and reorganizing the pieces of lives can be wise. The researcher discusses that an artwork rejuvenates one's life and works as self-renewal for bereft. An appropriate and timely guidance for such individual can be helpful. The dissertation research of Isis [14] revealed 4 prominent categories that are containment, repetition, storytelling and connectedness. It asserts that it facilitates women in regaining their lives.

Gulledge [15] shared that alike a deaf person uses sign language for communication; bereft have grief language. It has proposed that grief songs are helpful in reconciliation from denial phase. Furthermore, musical-lamentations can be useful that connects people with the world of deceased and give a space to mourn. Also, reflections that are shared with family and friends; helps in providing an emotional release [16]. Naturally, there are no definite verbatim that emulate mother's experience of loss. Here, an imperative helping role is to listen and guide them genuinely. Bowman [17] agreed that expressive arts such as talking and writing about grief are therapeutic. Speech can help make grief fit for human habitation. Human beings have strong urge to tell stories and through this, mothers can recall, recover and revive the cherished memories.

Thompson [18] puts in article that world seems changed when someone losses baby and it leaves an impression on relationship with others. It articulated that in grieving, women penetrate in a luminal sphere of thoughts and connect in acts of creation. When women experience loss, daily habits disrupts, activities associated with valued roles become dubious. Thus, expressive art within the therapeutic dyad can be used, and with small groups or communities of various kinds like talking circle, movement based warm-up like humming together etc. In addition, painting, creative writing, poetry, dialogue with the baby's image can be soothing [18]. Along with this, gardening can be therapeutic [13]. Similarly, mothers can have lock of hair or hand and foot print before deceased baby is cemented as per religious/cultural norms [17]. Nurses' knowledge and understanding of cultural and religious differences may facilitate bereaved parents. Further, Hochberg [19] discussed that connectedness can be observed when parents have babies' photographs with them. Thanatologists studied and shared that photographs are useful for grieving parents as they have value attached to it and are 'emotional touch stones and mementos' for families. Further, many hospitals have made the part of their protocol. Unobtrusively, people who work in such environment could share their experience when they try to create photographs that gently link the memories and feelings pertaining to the brief time that is spent with the baby and family. Gough [20] termed it as 'Photo Therapy' and told that it is a fascinating approach, collectively with sound therapeutic skills can bring warmth, depth and healing to bereaved parents. Since late 1970's, clinicians noted that photographs work as memento for bereft. Adding, copies of ultrasound scans can be momentous too.

Through the above-mentioned strategies, women can be helped. However, nurses and doctors are also in a position to help the victim via prescribing and administering medications as per clinical symptoms. These clinical practices require extensive trainings and up-to-date knowledge. NHS [13] suggests that antidepressants help in coping for shorter duration of time, as it is contention that it may interfere with the natural grieving process. While psychotherapy and counselling are in progress, antidepressants can be prescribed. NHS [13] also suggestively explains that sleeping tablets may also be prescribed for shorter duration to help mothers. However, these steps need coordination with psychiatrist and psychologist. as one doctor's advice may not be sufficient. Supporting, SIGN [21] discusses management via pharmacological, physical and psychosocial methods. The guideline talks about trans-dermal estrogens (with cyclical progestogen) which are more effective in moderate 


\section{Global Journal of Reproductive Medicine}

to severe post-natal depression. However, concerns about side effects, predominantly endometrial hyperplasia and thrombosis may limit its use. SIGN [21] discusses another randomized controlled trial of the use of anti-depressant therapy such as fluoxetine combined with at least one session of modified CBT (cognitive behavioural therapy), found useful. Another case study described in the research article, informs that both SSRIs (Selective serotonin reuptake inhibitors) and tricyclic antidepressants are effective in post-natal depression. On the contrary, CBT is as effective as antidepressants in alleviating depressive symptoms. It helps in changing unhelpful thoughts, beliefs and behaviours. Apart from this, physical exercise and its role in reducing levels of depression, has high-quality indication. The psychosocial management does include counselling and psychotherapy.

Counselling provides clarified ways ofliving with a better sense of well-being. Researches proves that systemic interventions are based on non-directive counselling such as, supportive listening for about few sessions by trained professionals; is an effective way to help bereaved women. Numerous studies have shown that family-focused interventions like couple involvement, focusing on parenting and their reactions to loss may help them relieve their depressive syndromes. However, controversial point that Hoy [6] shares is, that the divorce rates in married couples with deceased babies is in increasing trend varying from $40 \%-70 \%$ and at the same time some studies have even indicated divorce rate lower among bereaved parents. Counsellors' work is to help the chaotic families by facilitating in over-coming stress.

The bereavement healing programs are found to be helpful such as mentioned in the In God's Arm programme [22]; Saint Joseph regional medical centre; South Bend, Indiana is helping families to grieve and give options to bury the baby and facilitate support-group activities. In the booklet of NHS [13] it is discussed, that when babies are lost via early miscarriages; body is not as formed as of the mature babies and therefore, pain of lack of formed body presumably makes it harder to accept that the baby is dead. It asserts that the interment is an essential part in the process of bereavement, which is perhaps the expression of feelings and a way to remember the deceased. Some practices are observed by lightning candles and praying for the demise. It is imperative to know that the postponement of ceremonial moment may hinder healing and recovery. Mothers can be despondent and deeply distressed, if situations are not handled sensitively. They may cry uncontrollably or experience sharp pangs of grief, ruminative thoughts of deceased, yearning and loneliness [23]. At other times, a trivial event may provoke deep upset. Mother might exhibit aggression towards healthcare team and might feel guilty for being responsible for the deceased. Health- care teams need to be aware that grieving process is a gradual process and subsides progressively. Thus, in that situation, re assuring and listening to mothers is quite significant. Understandably, grieving is subjective and differs from person to person; it is essential to acknowledge it, as it helps in recovery. In NHS [13], it is discussed that, as time passes; the pain gradually lessens and it may be possible to consider future planning.

The care components need to be integrated and thus coordinated care-services are must to approach. From admission to discharge process, referrals and follow-up appointments, multidisciplinary approach with expert health professional's role is mandatory. Firstly, it is to find services that work separately which can be merged, to have holistic approach. Like, in hospitals where psychiatric ward and maternity ward are at different corners, consultations and referral to such services can be liaison. Secondly, role of patient care-coordinators, clinical nurse instructors and specialists' collaboration with physicians is extremely important. The study, Service framework for mental health and Wellbeing [1] discusses a regional model, which comprises of components such as identification, holistic assessment and integration of services, coordination of care, end of life and bereavement care, professional and public awareness. It is essential that care must engrossed principles of equality, accessibility, respect and dignity, empowerment and nonjudgmental approach. These initiatives are a step forward for patient-education, quality initiatives in sustaining best practices and influencing policy and commissioning. Furthermore, such initiatives can be replicated by under-developed countries.

The national agencies or charity based institutes reference here are not for promotional gains, but to have an information as a nurse to refer the clients and to get our hospitals liaise with. Some those are in UK and Pakistan; shall be considered in this paper. Bereaved Parents' Support Co-coordinators Kath \& Mike [24] works in a registered charity of England, Wales and Scotland; helps to find hope, healing and peace. They put in touch with one of their trained Bereaved Parents' Support befinders. Our physicians can liaise with them and direct parents to such charity support programmes. In Pakistan, secondary hospitals can refer cases to tertiary care settings at reasonable physician fee.

Moreover, Child Death Helpline [25] states that some helplines can be useful for parents who suffered by the death of a child at any age, from pre-birth to adult. Bereaved parents usually volunteer such help-lines and thus people can be referred there. It is important that if hospitals are referring families to such charitable institutes; must make sure that all volunteers are trained, supervised and supported by professional teams. In Pakistan, where health status and trained health-workers are not as approachable as in developed nations, such volunteers can be a guide to bereaved families. Further, multi-disciplinary groups can be formed. Several works have been done and are under process as well, for its awareness at professionals' level. Explicitly explained in The Promoting Mental Health Strategy \& Action Plan 2003-2008 [26] that working on strengthening communities and reducing structural barriers to mental health 


\section{Global Journal of Reproductive Medicine}

is fundamental. Thus, government should impart multi-agencies to have accessible and approachable care.

Gauthier et al. [27] researched 3 factors: parental selfefficacy, anxious attachment to a significant others and parental autonomous motivation that falls under SDT (Self-determination theory); which has certain needs identified: competence, relatedness and autonomy as a steer to help woman and her husband. This framework discusses the need for 'competence', which desires to interact effectively with the environment. Other, 'relatedness' refers to the need for close and secure emotional bonds and to feel loved and connected to significant others. Lastly, 'autonomy' need refers to the need to experience choice and the feeling that one is the initiator of one's actions, and to act in a way that is coherent with one's value. Firstly, 'parental self-efficacy' scale measures beliefs about skills of mother-hood. Practitioners need to have proper history of woman's parity and so it will help in smooth transition. Secondly, it is anxious 'attachment to a partner'. Thirdly, 'parental autonomous motivation' is composed of 5 motivation posited by SDT: intrinsic motivation (e.g. for the pleasure of seeing my child grow), identified motivation (e.g. having a child is a way to realize my life plan), introjected regulation (e.g. to please my social network- partner, family, friends), external regulation (e.g. to give new direction to my relationship with my partner) and motivation (e.g. I used to have good reasons for having a child, but now I wonder why). These frameworks are useful to assess needs and to help them in transition phase. Unless, healthcare professionals are aware, help in such cases remains sub-optimal.

Mental-health problems are associated with abortions, psychological barriers of birthing experiences and post-natal depression. Thus, our target needs to be in the same direction so that maternal deaths can be prevented [27]. Anderson [28] shares that losing a baby is linked to increased mortality. Mothers who experience stillbirth or lose a child in the first year of life, are 4 times likely to die in following decade than those who do not. The use of drugs and alcohol are found to be one of the causes of death in these women. A suggestive strategy is put forward by NHS [13] which says, that like other humans, bereaved women needs to take care of themselves. This can be possible through taking healthy food, practicing exercise and by being content with whatever life has offered [29].

\section{Conclusion}

To summarize, if bereavement is not inclined to positive side, it gives negative influence on women's health. Adding, it had been illustrated that depression in perinatal phase or prior to that, could lead to miscarriages and eventually disturbed psychological and psychosocial health. Through multidisciplinary teamwork, strategies and management can improve health status of women. Moreover, it is depicted that referrals can be made to psychiatrists who can prescribe medications, extensive counselling and psychotherapy. In addition, patient education pamphlets, related articles and community referrals to support-groups need to be strengthened. Follow-up appointments from hospitals can be tracked. Moreover, guidance towards post-partum stress-support group and call-centre support can be given for smooth transition to parent-hood.

It has alleged that the increased attention in implementing policies and protocols will reduce MMR. WHO [3] puts forward that the efforts need to be expanded in order to accelerate improvement towards reducing the disparity between developing and developed countries. It is of prime importance that all countries must have the clinical practice guidelines and policies such as CEMACH for vulnerable group. The outcome of these policies should focus on preventing, detecting and reducing the number of mortalities and morbidities. Moreover, timely intervention with the help of multi-disciplinary groups, stafftrainings and appropriate staff coverage in maternity services are needed to incorporate.

\section{References}

1. Weindling AM (2003) The Confidential enquiry into maternal and child health (CEMACH): A review of the history of confidential enquiries. BMJ 88(12): 1-6.

2. CMACE (Centre for Maternal and Child Enquiries) (2011) Saving Mothers' lives: Reviewing maternal deaths to make motherhood safer: 2006-2008. The Eighth Report of the Confidential Enquiries into Maternal Death in the United Kingdom. BJOG 118(Suppl 1): 1-203.

3. WHO (World Health Organization) (2010) Trends in Maternal Mortality: 1990 to 2008. pp. 1-55.

4. CEMACH (Confidential Enquiry into Maternal and Child Health) (2007) Saving Mothers' lives: Reviewing maternal deaths to make motherhood safer: 2003-2005. The Seventh Report of the Confidential Enquiries into Maternal Death in the United Kingdom. BJOG pp. 1-296.

5. CLIMB (Centre of Loss in Multiple Birth) (2003) The death of your twin or triplet babies. A pamphlet for parents and families who have experienced the death of both or all of a set of twins, triplets or higher multiples, during pregnancy, at birth, or in infancy pp. 1-4.

6. Hoy G (2011) Unveiling the myths in the truth about grief.

7. Nikcevic A, Tinkel S, Kuczmierczyk A, Nicolaides K (2005) Investigation of the cause of miscarriage and its influence on women's psychological distress. British Journal of Obstetrics and Gynaecology 106(8): 808813.

8. DHSSPS (Department of Health, Social Services and Public Safety) (2010) Service framework for mental health and wellbeing.

9. Protect life (2006) The Northern Ireland Suicide Prevention Strategy and Action Plan 2006-2011. Investing for health. Department of health, social services and public safety.

10. Ritsher JB, Neugebauer R (2002) Perinatal Bereavement grief scale: Distinguishing grief from depression following miscarriage. Assessment 9(1): 31-40.

11. Le N, Boyd R (2006) Prevention of major depression: Early detection and early intervention in the general population. Clinical Neuropsychiatry 3(1): 13

12. Glavin K (2011) Screening and prevention of postnatal depression. Diakonova University College, Oslo, Norway, pp. 1-20.

13. NHS (2006) Talking about bereavement. Health Scotland. 


\section{Global Journal of Reproductive Medicine}

14. Isis P (2003) The impact of the expressive therapies on adult grief. Expressive arts in bereavement; case studies. The Forum. An International, Interdisciplinary Organization 29(2): 10.

15. Gulledge D (2003) Grief in Motion. Expressive arts in bereavement; case studies. The Forum. An International, Interdisciplinary Organization 29(2): 10.

16. Strickland A (2003) The healing power of music in bereavement Expressive arts in bereavement; case studies. The Forum. An International, Interdisciplinary Organization 29(2): 4.

17. Bowman T (2003) Using literary resources in bereavement work: evoking words of grief. Expressive arts in bereavement; case studies. The Forum. An International, Interdisciplinary Organization 29(2): 9.

18. Thompson B (2003) The expressive arts and experience of loss Expressive arts in bereavement; case studies. The Forum. An International, Interdisciplinary Organization 29(2): 1.

19. Hochberg T (2003) Touching souls: healing with bereavement photography. Expressive arts in bereavement; case studies. The Forum. An International, Interdisciplinary Organization 29(2): 6.

20. Gough M (2003) Photo Therapy with the Bereaved. Expressive arts in bereavement; case studies. The Forum. An International, Interdisciplinary Organization 29(2): 7.
21. SIGN (Scottish Intercollegiate Guidelines Network) (2002) Postnatal Depression and Puerperal psychosis: A National clinical Guideline no. 60.

22. In God's Arm (2008)

23. Neimeyer R, Prigerson H, Davies B (2002) Mourning and Meaning. American Behavioural Scientists 46(2): 235-251.

24. Kath \& Mike (2012) Care for the Family.

25. Child Death Helpline (2010).

26. (2003) Promoting Mental Health Strategy and Action Plan 2003-2008 Department of Health, Social Services and Public Safety.

27. Gauthier L, Guay F, Sen écal C, Pierce T (2010) Women's Depressive Symptoms during the transition of Motherhood: The role of Competence, Relatedness and Autonomy. J Health Psychol 15(8): 11451156 .

28. Anderson P (2011) Loss of a baby linked to increased mortality in parents. Medscape Nurses news.

29. (2010) Service framework for mental health and wellbeing. Department of Health, Social service and Public Safety.

Your next submission with Juniper Publishers will reach you the below assets

- Quality Editorial service

- Swift Peer Review

- Reprints availability

- E-prints Service

- Manuscript Podcast for convenient understanding

- Global attainment for your research

- Manuscript accessibility in different formats

( Pdf, E-pub, Full Text, Audio)

- Unceasing customer service

Track the below URL for one-step submission https://juniperpublishers.com/online-submission.php 